\title{
Conjunctival Melanoma in situ arising over conjunctival nevus-a case report.
}

\author{
Dr. Nameirakpam Mamota ${ }^{1}$, Dr. Ibohal Salam ${ }^{2}$ \\ ${ }^{I}$ (Department of Ophthalmology, Regional Institute of Medical Sciences, Imphal) \\ ${ }^{2}$ (Department of Ophthalmology, Regional Institute of Medical Sciences, Imphal)
}

\begin{abstract}
We report here a case of 38 years old healthy male, who presented in the eye OPD with blackish mass over conjunctiva which was increasing in size since lyear. On local examination the mass was on the bulbar conjunctiva near the limbus, darkly pigmented, $0.3 \times 0.3 \mathrm{~cm}$ size, irregular margins with uneven surface and mobile over the sclera. Other ocular examinations were normal. General investigations were done. The mass was excised under peribulbar local anaesthesia and sent for histpathological examination. The report showed moderate to severe nuclear atypia with evidence of pategoid spread. The subepithelial fibro collaginous tissue shows focal areas with moderate lymphocytic infilteration. There is no evidence of stromal invasion in any of the sections studied. The features were suggestive of malignant melanoma in situ. Conjuctival melanoma is a rare unilateral tumour and account for approximately $2 \%$ of the malignancies of the eyes. We conclude that early surgical excision of nevus is beneficial as it lower the risk of metastatic disease and tumor associated mortality.
\end{abstract}

Keywords: conjunctival mass, nevus, conjunctival melanoma.

\section{Introduction}

Conjuctival melanoma is a potentially fatal ocular surface tumour affecting about 0.2 persons per million and representing approximately 2 percent of the malignancies of the eye. It originates de novo in about 5 percent of all cases, from preexisting conjunctival primary acquired melanosis (PAM) in 75 percent, and from nevi in 20 percent. ${ }^{1,2}$ The purpose of reporting this case is because of the rarity of its occurrence and associated poor prognosis with late diagnosis of the malignancy.

\section{Case History}

A 38 years old healthy male came to eye opd with a chief complaint of ocular discomfort and a blackish mass over conjunctiva of left eye. The patient noticed the blackish mass during his adulthood days. The mass was initially small and gradually increase in size over a period of 1 year according to the patient. It was painless with no history of trauma. No similar history in the past. Family history and personal history were not significant.

Ophthalmological examinations included the vision, local examination of the eye using torch illumination, slitlamp and fundus ophthalmoscopy, and intraocular pressure measurement.

The patient visual acuity was $6 / 6$ in both eyes on Snellel charts. On slit lamp examination of left eye conjunctiva, there was a dark brown pigmented lesion, $3 \mathrm{~mm}$ from the limbus in the bulbar conjunctiva,elevated, nodular with irregular margins, uneven surface and moves freely over the sclera. The cornea, anterior chamber, iris and pupil were all within normal limit in both eyes. Fundus examination of both eyes was normal. Intraocular pressure was $13 \mathrm{mmHg}$ in both the eyes by goldmann applanation tonometer. Regional lymph nodes were not palpable.

\section{Investigations}

On investigation, his blood parameters including complete hemogram, liver and kidney function tests and blood sugar were all within normal limit. Chest X-Ray and ECG showed no significant pathology.

\section{Management}

A provisional diagnosis of conjuctival nevi with differential diagnosis of melanoma and squamous cell carcinoma were considered. The patient was referred for medical clearance.

The mass was then excised completely with a wide margins from the apparent lesion under peribulbar local anaesthesia . The conjunctival defect was then closed with 8-0 ethilon. The excised conjunctival nevus was then sent for histopathological examination. The following features were seen in histopathlogical examination : 1. The studied sections show tissue lined by stratified squammous keratinised epithelium, many of which display increased in melanocytes involving the entire thickness of the epithelium. 
2. Individual cells display moderate to severe nuclear atypia with prominent eosinophilic nucleoli..

3. Abnormal mitoses are seen.

4. Evidence of pategoid spread is seen.

5. The subepithelial fibrocollagenous tissue shows focal areas with moderate lymphocytic infiltration. There was no stromal invasion seen in any of the section studied.

These features establish the diagnosis of conjunctival melanoma in situ.

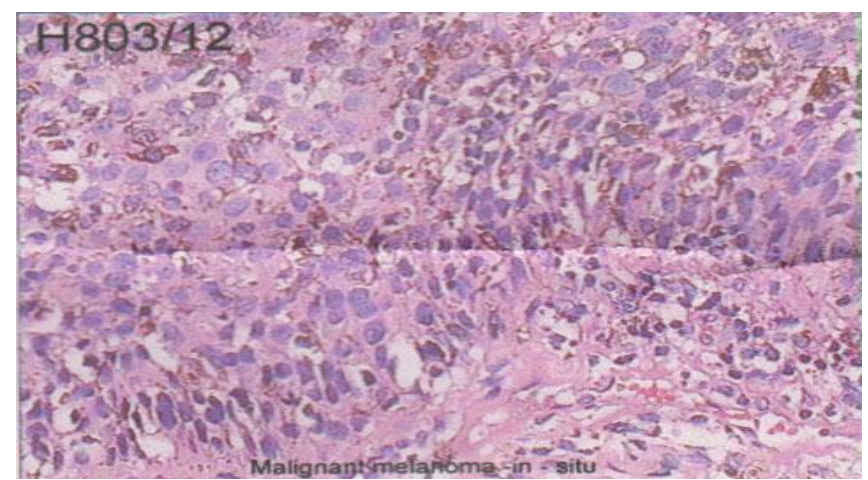

Fig 1: Histopathology picture of malignant melanoma in situ

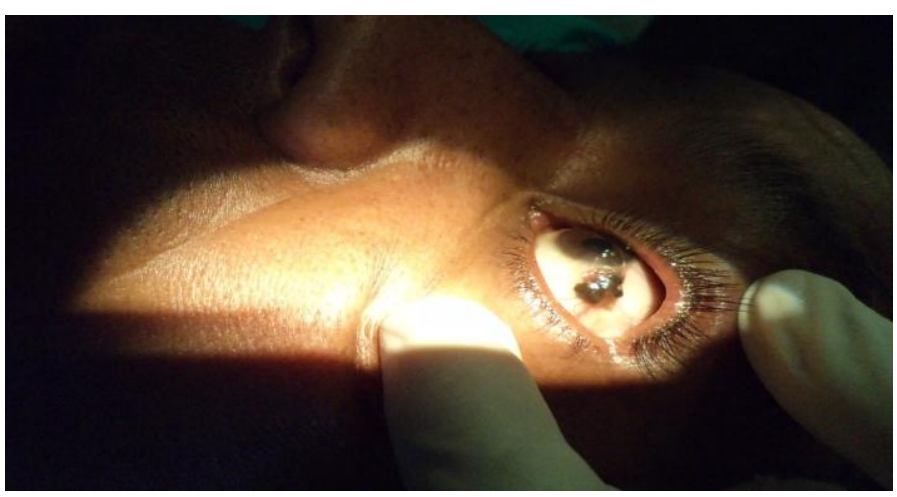

Fig 2: Pigmented nodular tumour at the limbus

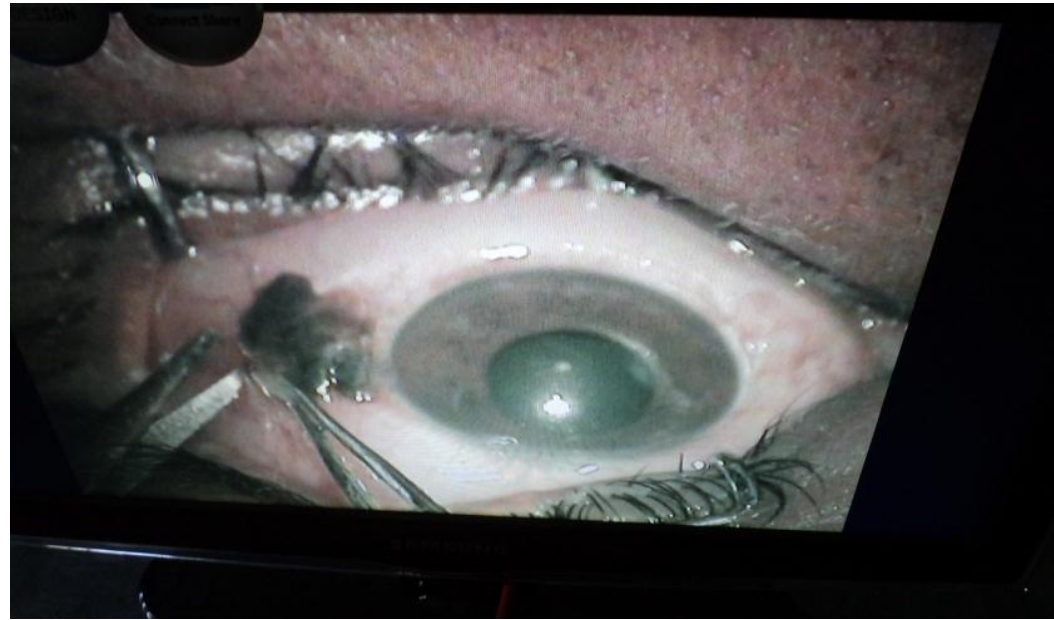

Fig 3: Surgical excision of the mass. 


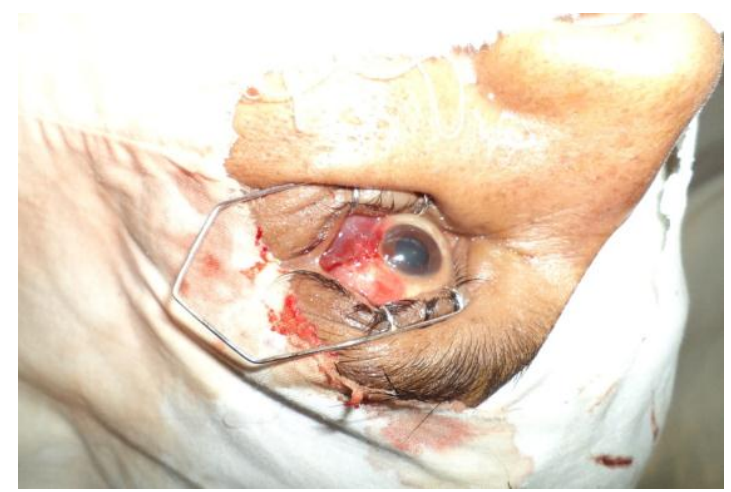

Fig 4: Closure of conjunctival defect

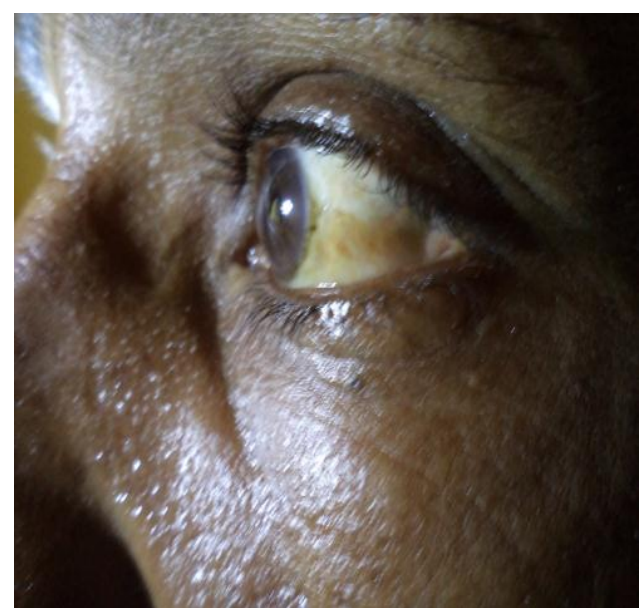

Fig 5: Follow up after 2 months

\section{Discussion}

Conjunctival melanoma is a rare unilateral tumour with a mortality rate of 23 to $30 \%$ in 10 years. ${ }^{1,2}$ The disease is more common in middle aged and elder persons between $4^{\text {th }}$ to $7^{\text {th }}$ decades of life. ${ }^{3,4}$ It may arise anywhere in the conjunctiva. Its spread is made by the lymphatics affecting first the preauricular nodes and then submandibular and cervical nodes. Tumours that are not on the bulbar surface appears to behave more aggressively and are viewed as suspicious for melanoma and excised. ${ }^{5}$ Excisional biopsy is the only way to differentiate melanoma from benign lesion. In the present case, the excised conjunctival nevus was sent for histopathologic examination and a definitive diagnosis of melanoma was made based on the findings.

Histologically four types of atypical melanocytes are seen: small polyhedral, spindle, ballon, and round epitheloid cells with eosinophilic cytoplasm. ${ }^{6}$ Immunohistochemical stains such as HMB-45 or Melan-A can demonstrate melanocytes. The presence of pategoid spread, mixed cell tumours versus spindle cell tumours, histologic evidence of lymphatic invasion, high number of mitoic figures and a high cell proliferation index predicts and adverse prognosis. ${ }^{1,2}$ The primary treatment is surgical excision of the entire tumour with wide surgical margins of $3-5 \mathrm{~mm}$. Areas of adjacent PAM (primary acquired melanosis) should also be treated by surgical excision, cryotherapy, brachytherapy or topical chemotherapy using mitomycin $\mathrm{C}^{7}$ Exenteration is indicated once the orbit is involved. Prognosis is poor and life expectancy short for those associated with regional lymph node metastasis. ${ }^{8}$

\section{Conclusion}

Conjunctival melanoma is a relatively rare malignancy. Being a rare clinical entity it should be considered on the differential diagnosis of pigmented eye surface lesions, especially when growing on preexisting tumours such as PAM or nevus. Timely diagnosis of melanoma is essential because of their high lethal potential. Early surgical excision of benign conjunctival nevus turning into malignant melanoma can potentially save the patient from future morbidity and mortality of conjunctival malignant melanoma. 


\section{REFERENCES}

[1.] Seregard S. Conjunctival melanoma. Surv Ophthalmol. 1998; Jan-Feb;42(4):321-50.

[2] Folberg, R, Mclean IW, Zimmerman LE. Malignant melanoma of the conjunctiva. Hum Pathol 1985;16(2): 136-143.

[3]. De Woof- Rouendaal D. Conjunctival melanoma in the Netherlands:a clinico-pathological and follow -up study. [Thesis ] Katwijk, All in BV,1990.

[4] Seregard S, Kock E. Conjunctival melanoma in Sweden 1961-91. Acta Ophthalmol 1992;70:289-296.

[5] Tuomaala S, Kivelä T. Metastatic pattern and survival in disseminated conjunctival melanoma: implications for sentinel lymph node biopsy. Ophthalmology. 2004 Apr; 111 (4):816-21.

[6] Jakobiec FA, Folberg, R, Iwamoto T. Clinicopathologic characteristics of premalignant and malignant melanocytic lesions of the conjunctiva. Ophthalmology 1989; 96: 147-166.

[7] Shields JA, Shields CL, dePotter P. Surgical management of conjunctival tumours. The 1994 Lynn B, McMahan Lecture. Arch Ophthalmol 1997; 115: 808-815.

[8] Tatla T, Hungerford J, Plowman $\mathrm{N}$ et al. Conjunctival melanoma: The role of conservative surgery and radiotherapy in regional metastatic disease. Laryngoscope 2005; 115: 817-822. 